\title{
Osteogenic activity of lactoferrin and its application in contemporary dentistry
}

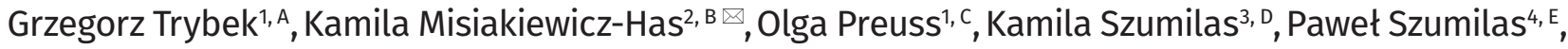 \\ Magda Aniko-Włodarczyk ${ }^{1, F}$, Katarzyna Grocholewicz ${ }^{5, G}$ \\ ${ }^{1}$ Pomeranian Medical University in Szczecin, Department of Oral Surgery, Powstańców Wlkp. 72, 70-111 Szczecin, Poland \\ 2 Pomeranian Medical University in Szczecin, Department of Histology and Embryology, Powstańców Wlkp. 72, 70-111 Szczecin, Poland \\ ${ }^{3}$ Pomeranian Medical University, Department of Physiology, Powstańców Wlkp. 72, 70-111 Szczecin, Poland \\ ${ }_{4}^{4}$ Pomeranian Medical University, Department of Social Medicine and Public Health, Żołnierska 48, 71-210 Szczecin, Poland \\ ${ }^{5}$ Pomeranian Medical University, Department of Integrated Dentistry, Powstańców Wlkp. 72, 70-111 Szczecin, Poland \\ A ORCID: 0000-0002-3900-4901; ${ }^{B}$ ORCID: 0000-0001-7880-7507; ${ }^{C}$ ORCID: 0000-0002-9744-8885; ${ }^{D}$ ORCID: 0000-0002-5635-1630; \\ E ORCID: 0000-0002-3328-7904; F ORCID: 0000-0002-3342-0605; ${ }^{G}$ ORCID: 0000-0002-1468-4664 \\ $\triangle$ kamila.misiakiewicz@pum.edu.pl
}

\begin{abstract}
Introduction: Lactoferrin (Lf) is a protein in the transferrin family with many biological functions. One novel activity of lactoferrin described recently is its regulatory function in bone morphogenesis. Lactoferrin has been shown to promote the growth, development, and differentiation of osteoblasts as well as to decrease osteoclast survival. Lactoferrin receptors (LfRs) mediate the multiple functions of lactoferrin. This review focuses on LfRs associated with bone and the intestines. The best known LfR is small intestine LfR (intelectin), which facilitates iron absorption and iron metabolism in humans. Many data from in vitro and in vivo studies have indicated that lactoferrin
\end{abstract}

\section{INTRODUCTION}

Lactoferrin (Lf) is a nonheme iron-binding multifunctional protein of the transferrin family that plays a variety of functional roles, showing immunomodulatory, anticancer, antibacterial, and antiviral properties $[1,2,3,4]$. As a biologically active protein, Lf exerts broad physiological effects on different types of cells, including bone cells [5, 6], via specific receptors. One novel activity of Lf that has been recently demonstrated is its regulatory function in bone morphogenesis and bone homeostasis [7]. Lactoferrin has been shown to promote the growth, development, and differentiation of osteoblasts (OBs) [8] as well as to decrease osteoclast survival $[9,10]$. Due to Lf's osteogenic activity, many in vitro and in vivo studies have been performed to seek innovative methods to create biomaterials with Lf, in order to reduce the local imbalance in bone homeostasis occurring in osteoporosis and other bone diseases. The aim of this study was to present the osteogenic activity of lactoferrin and ways of applying in oral surgery.

\section{LACTOFERRIN RECEPTORS}

Lactoferrin receptors (LfR) mediate the multiple functions of Lf. These receptors seem to differ among species, tissues, cell types, and even degree of maturation. Reports have described promotes bone formation by increasing the proliferation of osteoblasts and the ability of cells to synthesize and mineralize the bone matrix. Lactoferrin additionally inhibits osteoclastogenesis, reducing the number of osteoclasts and thus bone resorption. Lactoferrin, with its numerous antimicrobial, anti-inflammatory, and also osteogenic properties has found a number of applications in contemporary dentistry, especially in dental surgery, in periodontology, and in pedodontics.

Summary: This review presents in vivo and in vitro studies demonstrating the osteogenic and anti-inflammatory activity of Lf and its practical application in oral surgery and dentistry. Keywords: lactoferrin; bone; oral surgery; dentistry.

various cells with LfRs; from the point of view of Lf's function on bone, the most important LfR are those associated with bone and with the intestines. Reverse transcriptase polymerase chain reaction (RT-PCR) studies reveal that LfR are expressed at high levels in the fetal small intestine and at lower levels in human intestinal epithelial cells line (Caco-2 cells). Small intestine LfR, also called intelectin, is the best known of the LfRs, and facilitates iron absorption and iron metabolism in humans [11]. Lönnerdal studied the presence of LfRs in the brush border membrane of infant rhesus monkey intestine and from fetal and infant human intestines. The binding of the receptor is specific to human and monkey Lf; bovine Lf (bLf) did not bind to the receptor. It has also been found that enzymatic deglycosylation does not affect the binding of human Lf to its receptor, so glycan is not needed for receptor recognition [12]. Since Lf is resistant to proteolytic digestion [13] and LfR is present in the intestinal brush border, uptake of Lf from the lumen to the blood is rapid [14].

Lactoferrin may also interact with heparan sulfate proteoglycans (HSPGs), glycosaminoglycans (GAGs), glycoprotein 330 (gp330), low-density lipoprotein receptor (LDLR), nucleolin, and the asialoglycoprotein receptor $[15,16,17,18,19]$. In fact, LfR's propensity to bind to most cells confers protection against infection by some viruses [20]. Besides, the low-density receptor-related protein/alpha 2-macroglobulin receptor (LRP/alpha $2 \mathrm{MR}$ ) and gp330 both may function as endocytosis-mediating 
receptors for a large number of ligands (including Lf) in different organs [19]. Bovine Lf has also been used to treat intestinal giardiasis when internalized by receptor-mediated endocytosis [21]. Of the various LfR, only low-density lipoprotein receptor-related proteins 1 and 2 (LRP1 and LRP2) are present on OBs [22]. The low-density lipoprotein receptor-related proteins 1 is at least partially responsible for LF's mitogenic effect on OBs through activation of LPR1-mediated p42/44 mitogenactivated protein kinase (MAPK) [22]. Surprisingly, induction of osteoblast survival by Lf is not dependent on LPR1 [23]. Hou et al. discovered that Lf induces IGF-1/IGF-1R expression in a concentration-dependent manner, and that it induces proliferation but inhibits apoptosis of OBs through mediation of IGF-1R [24].

Lactoferrin has beneficial effects on bone resorption by reducing the number of osteoclasts formed from precursor cells. Although effects of Lf on osteoclast development have been noted, Lf does not affect mature osteoclast activity [8]. Osteoclasts are unique cells whose hyperactivity is involved in bone pathologies such as osteoporosis and osteolytic diseases. Osteoclasts derive from a mononuclear phagocytic lineage that expresses cluster of differentiation 14 (CD14) on the cell's surface [25]. Factors such as hormones, cytokines, and growth factors that regulate osteoclast differentiation and activity act directly on osteoclasts and their precursors, or indirectly OBs to modify the expression of proteins that control osteoclastogenesis, or use a combination of both mechanisms, direct and indirect [26, 27]. The mechanisms involved in the effect of Lf on osteoclasts are not known, and the receptor responsible for mediation of Lf's effect on osteoclasts has not yet been discovered [28]; however, it may be associated with the indirect influence of Lf on bone metabolism through modulation of cytokine production [29].

\section{IN VITRO STUDIES}

Osteogenesis is a complex process that includes the differentiation of mesenchymal cells into preosteoblasts and OBs, leading to the synthesis and deposition of bone matrix proteins [30]. Data obtained from in vitro studies support the thesis that Lf has osteogenic activity. To examine the in vitro influence of Lf on osteogenesis, an undifferentiated mesenchymal cell line, or one isolated from bone marrow cells, was used. One such cell line are myoblast progenitor cells ( $\mathrm{C} 2 \mathrm{C} 12$ cells), which originate from undifferentiated mesenchymal cells; they have the capacity to differentiate not only into myoblasts, but also into OBs, chondroblasts, and adipocytes [31]. In experiment by Yagi et al., C2C12 cells were cultured in medium supplemented with Lf in increasing concentrations [32]. The addition of Lf resulted in the proliferation and differentiation of $\mathrm{C}_{2} \mathrm{C} 12$ cells into osteoblastic and chondroblastic lineages. It was noted that there was an increase in the activity of alkaline phosphate and increases in the expression of Runx2 (essential for OB differentiation and chondrocyte maturation) of Sox 9 (which regulates many developmental events, such as cartilage development), and of osteocalcin (OC), which plays an important role in bone turnover and formation. Muscle cell markers have also undergone reduction after the addition of Lf. Messenger RNA (mRNA) analysis indicated that all effects were exerted by LRP1 LfRs expressed in $\mathrm{C} 2 \mathrm{C} 12$ cells [32].

The effect of Lf on osteoblast-like cells has been investigated in animal and human OBs. The study Cornish et al. [8] make use of primary cultures of fetal rat OBs enzymatically digested from 20-day rat calvariae, human OBs from enzyme-treated normal trabecular bones (obtained from patients undergoing knee or hip arthroplasty), and human osteoblast-like cell line (SaOS-2). The cells were cultured in medium supplemented with Lf isolated from fresh bovine skim milk. Additionally, a model of osteoclast function was created utilizing cultures of bone marrow cells obtained from long bones of mice (aged 4-6 weeks) and rat osteoclasts isolated from the long bones of 1-day-old rats cultures. The study showed that bLf stimulated osteoblast proliferation and the differentiation of rat osteoblastlike cells, as well as of the SaOs-2 cell line and human OBs in primary cultures. Lactoferrin also reduces $\mathrm{OB}$ apoptosis and has the ability to inhibit osteoclastogenesis in mouse bone marrow cultures, while having no effect on bone resorption by isolated mature OBs. Those researchers concluded that Lf is a potential therapeutic target in some bone disorders [8].

The same proliferative effects of bLf on OBs was observed in the mouse osteoblastic clonal cell line $\mathrm{MC}_{3} \mathrm{~T} 3-\mathrm{E} 1$ in vitro. Supplementation of cultured medium with $100 \mu \mathrm{g} / \mathrm{mL}$ of bLf resulted in the stimulation of $\mathrm{OB}$ proliferation, and increased the length of the G2/M and S phases of the cell cycle [33]. It was established that the bLf can induce the proliferation of MC3T3-E1 OB cells by stimulating 3 members of the mitogenactivated protein kinases (MAPK): extracellular-signalingregulated kinase (ERK), c-Jun $\mathrm{N}$-amino-terminal kinase (JNK), and p38 kinase [34].

Recently, new biomaterials with recombinant human or animal Lf have been sought to promote osteogenic differentiation of cells in vivo, so as to maintain bone homeostasis. There have been trials to find such biomaterials by making a culture of mesenchymal stem cells (MSCs) isolated from rabbit bone marrow in the presence of hydroxyapatite (HA) nanocrystals with surface loaded bLf. The hydroxyapatite and Lf have been shown to induce MSC differentiation and enhance anabolic bone activity, and can be expected to be potential tools in bone regeneration. The coupling of HA and Lf has been shown to play a role in the induction of osteogenic markers, represented by the stimulation of alkaline phosphatase (ALP) activity and the expression of osteogenic-associated genes, such as RUNX2, $B M P 2$, SPARC [35].

The same in vitro model, HA nanocrystals with Lf was used to test the behavior of $\mathrm{OB}$ and osteoclast growth. The preosteoblast cell line $\mathrm{MC} 3 \mathrm{~T} 3-\mathrm{E} 1$, a model of $\mathrm{OBs}$, and the murine monocyte/macrophage cell line RAW 264.7 Osteoclasts (a model of osteoclastogenesis) were used in the experiment. The cells were cultured separately or cocultured in the presence of HA-Lf. The hydroxyapatite nanocrystals coupled with the bLf exerted a positive effect on the viability of OBs and decreased 
the percentage of apoptotic cells. Additionally, after 14 days of culture, HA-Lf significantly upregulated the expression of an osteoblast-specific transcription factor (Osterix) and of integrin-binding sialoprotein (IBSP) genes. The HA-Lf system reduced osteoclast formation and the expression of Integrin $(\operatorname{Itg} \beta 3)$. The crosstalk between OBs and osteoclasts has been evaluated in coculture. The HA-Lf and Lf induced a significant down-regulation in the expression of the osteoclast-associated immunoglobulin-like receptor (Oscar) and Catepsin $\mathrm{K}$ (CtsK) genes. The results reveal that $\mathrm{HA}$ and Lf act in synergy when coupled together as an anabolic factor for $\mathrm{OB}$ and bone matrix deposition, and as an inhibitor of osteoclast formation and activity. The authors suggested that the HA-Lf system could be used for several bone-related pathologies, and in future in tissue engineering and regenerative medicine [36].

A similar experimental model was created by Vandrovcova et al. to evaluate the effects of Lf in prepared collagen hydrogels. An artificial extracellular matrix (aECM), consisting of collagen type I fibrils, was formed in the presence of Lf at two different concentrations ( 0.5 and $1 \mathrm{mg} \mathrm{mL-1)}$ ). In this model, the effects of aECM on adhesion, growth, and osteogenic differentiation of human osteoblast-like Saos-2 cells were investigated. The greatest numbers of cells were found in cultures with collagen-Lf coating (particularly with higher concentrations of Lf), which produced higher levels of osteocalcin. These authors concluded that Lf promotes adhesion, growth, and osteogenic differentiation of Saos-2 cells, and could be used as a component of bone implant coating [37].

Another promising model was made with the use of the type-1 collagen gel used as a culture system in which OBs were grown in a 3D structure, affecting cell shape, cell-cell interaction, and response to soluble factors [38, 39, 40]. In the study by Matthews et al. (2014), primary calvarial OBs isolated from male and female rats and murine preosteoblastic MC3T3-E1 cells were cultured in 3D type-1 collagen gels with the medium supplemented with growth factors inducing osteoblast proliferation (bLf, TGF- $\beta$, and PDGF) [40]. Increasing concentrations of lactoferrin induced a dose-dependent increase in the proliferation of cells. Cell differentiation was determined by mineral staining and osteocalcin, bone sialoprotein, alkaline phosphatase, and dentin matrix protein gene expression analysis. The cells grown in 3D gels showed positive mineral staining and the induction of OB marker genes earlier than did cells grown in a $2 \mathrm{D}$ culture (on plastic) [40]. On the other hand, the effect of bLf on OB-induced extracellular matrix (ECM) calcification was also studied [41] with human osteosarcoma-derived MG63 cells as a model. The cells were cultured in plates coated with type I collagen in medium supplemented with bLf. The culture of the cells under osteogenic conditions resulted in significant stimulation of ECM calcification, accompanied by significant promotion of osteocalcin production in the late stage of the osteogenic process (week 3), and in a significantly elevation of ALP activity. The results obtained, with the influence of bLf on MG63 cells, suggest that a type I collagen membrane is useful as a drug delivery carrier for Lf in bone tissue engineering [42]. As was shown in a biochemical and biophysical studies [43], recombinant human Lf developed from brown rice grains [44] was similar to native human Lf (nhLf). Primary rat OBs (using the procedure of Cornish et al. [8]), cultured with the supplementation of holo-rhLf in increasing concentrations, showed a significant increase in cell growth at every concentration of rhLf [43].

Lactoferrin is a component of human secretions, including saliva $[1,4,45]$; it is postulated to favor repair mechanisms by inducing cell differentiation [46]. For this reason, its timedependent influence on osteoblast-like cell morphology and proliferation capacity was assessed. Murine $\mathrm{MC}_{3} \mathrm{~T} 3 \mathrm{OBs}$ were cultured in a medium containing human saliva collected from healthy volunteers through stimulation. The presence of saliva in the medium significantly reduced the proliferation capacity of $\mathrm{MC}_{3} \mathrm{~T}_{3} \mathrm{OBs}$ and resulted in the downregulation of key marker genes of OB differentiation, such as RUNX2, osteocalcin, ALP, alpha-1 collagen (Colla1). However, the cultured media contained active salivary enzymes (alpha-amylase, lysozyme, peroxidase, and collagenase), which harm OB-like cells. It was therefore recommended that the clinical contact period of saliva and bone should be shortened as much as possible [47].

\section{IN VIVO STUDIES}

In vivo experiments have also been performed to confirm the osteogenic function of Lf documented in the in vitro studies. In a murine model, the effects of bLf were tested with increasing doses of Lf, given as daily injections over the periosteum of the right hemicalvaria in normal adult mice for 5 days. The bLf produced increased new bone formation [8]. No positive effect of Lf on the amount of new bone formation was observed in experimentally induced bone defects in rabbit calvaria $[48,49]$. Histological and histomorphological analyses indicated that sites filled with and without the addition of bLf were associated with the formation of a mixture of woven and lamellar bone, although the differences between the groups were not statistically significant with regard to the amount of new bone formation [49]. The authors concluded that although there was no significant difference between the tested groups, it seemed that the addition of LF increased bone formation [48, 49].

The properties of Lf in the regeneration of bone have been confirmed in a similar study performed in adult male Fischer rats [50]. Non-critical-sized calvarial bone defects $(2.7 \mathrm{~mm})$ were trephined into the dorsal bone of both sides of a midsagittal suture. An absorbable collagen sponge was placed in the bone defects. After that, the animals were treated with Lf injected intraperitoneally. Histological and histomorphometric examination after 4 weeks showed that, in both Lf-treated animals, new bone was formed and OB-like cells were observed around the bony rims. In the control animals, the absorbable collagen structure had been absorbed, and the defects had filled with dense fibrous connective tissue, with minimal new bone formation. Additionally, there were more OB-like cells in the Lf-treated groups than in the control group [50]. 
To assess the effect of Lf on bone regeneration during distraction osteogenesis, an experiment was performed using unilateral osteodistraction in the right tibia of male rabbits. The experimental group of rabbits was treated with bLf $(85 \mathrm{mg} / \mathrm{kg} /$ day) orally from the commencement of the distraction until the end of the experiment. The results from radiological and histological examinations ( 4 and 8 weeks of treatment) showed that bLf treatment resulted in earlier bone mineralization and better new bone formation in the distracted callus. In the serum of the rabbits, the level of bone alkaline phosphatase (BALP) was higher. The results also showed the increased expression of osteoprotegerin (OPG) mRNA (OPG inhibits osteoclast differentiation and suppresses osteoclast bone resorbing capabilities) and a decreased level of receptor activator for nuclear factor kappa-B ligand (RANKL) mRNA in the distracted calluses. Immunohistochemical examination showed an increase in the number of OPG-positive cells without any difference in the number of RANKL-positive cells in the calluses. These results pointed to a decrease in the RANKL/OPG ratio (the key regulatory system of bone resorption). The authors suggested that treatment with bLf could promote bone regeneration during distraction osteogenesis in rabbits, and that the OPG/RANKL/ RANK system might be a major mechanism for increased bone formation and decrease bone resorption [51].

The osteogenic activity of bLf was also confirmed in our study (unpublished data), in which new bone formation in experimentally induced defects in a rabbit frontal bone was observed after a month. Bovine Lf was introduced orally in the form of an aqueous solution of freeze-dried colostrum (bovinum Colostrigen Genactiv, Poland) at $100 \mathrm{mg} / \mathrm{kg}$ bw/day (Fig. 1).

A similar mechanism for improving bone mass and microstructure using bLf was observed in an ovariectomized (ovx) rat model [52]. The ovx 6-month-old virgin rats were treated with increasing doses of bLf for $6 \mathrm{x}$ months. One group of ovx rats was supplemented with E2 as a positive control. After the treatment, the femur and the L2-4 vertebrae were analyzed. Ovariectomized significantly reduced bone and tissue volume, trabecular number and thickness, and increased trabecular

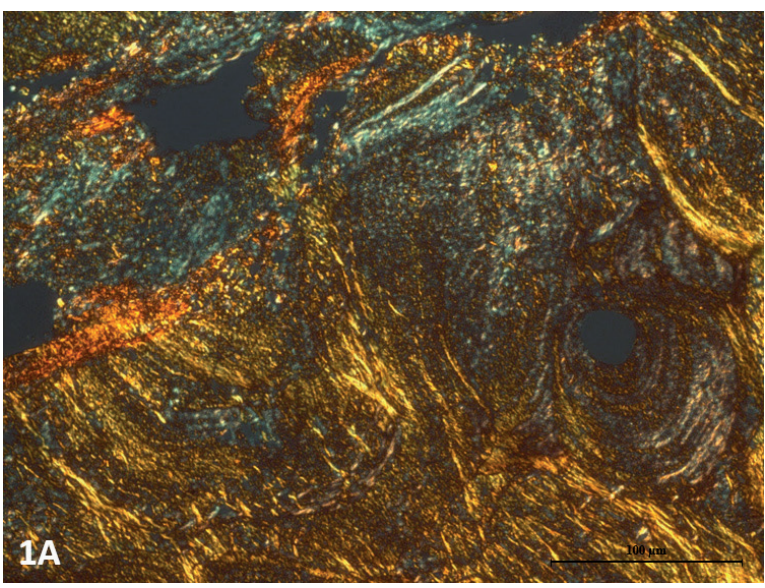

separation in both the femur and vertebrae. Treatment with bLf or estradiol protected the bone from ovx-induced effects. The bone mineral density (BMD) volumes increased in the bone of rats treated with higher doses of bLf, while lower doses of bLf did not significantly alter BMD. Higher doses of Lf affected the turnover of serum biochemical markers of the bone through significantly increasing levels of osteocalcin and BALP, and decreasing levels of C-telopeptide of the beta 1 chain of type-1 collagen of bone (B-CTx) and of collagen type-1 N-telopeptide (NTX). Additionally, the expression level of RANKL mRNA in the proximal femur was decreased, but the expression of OPG mRNA increased after treatment with higher doses of Lf. The RANKL:OPG mRNA ratio in the bone showed a significant decrease in animals treated with Lf in higher doses, while no changes were observed at the lowest dose of Lf [52].

The effects of Lf on bone physiology have already been studied in an osteopenic animal model. Ovariectomized rats were treated orally with bLf in increasing doses for 3 months. Treatment with bLf protected against reduction in bone volume, trabecular number and thickness, and an increase in trabecular separation. Treatment with Lf also resulted in substantial protection against loss of bone mass density and increased mechanical strength. Analysis of biochemical markers of bone remodeling (serum Ca and osteocalcin) indicated greater bone formation and reduced bone resorption. Bovine Lf in higher doses also led to a significant decrease in calcium and significantly elevated the level of serum calcitonin, while suppressing production of serum tumor necrosis factor- $\alpha$ (TNF- $\alpha$ ) and interleukin 6 (IL-6) [53].

Blais et al. used ovx $\mathrm{C}_{3} \mathrm{H}$ mice and sham-operated mice in an in vivo animal model of postmenopausal osteoporosis. It was shown that supplementing the control diet with bLf improved bone mineral density and femoral failure load in a dose-dependent manner [9]. The improvement in bone status caused by bLf could have been mediated by modulation of the immune function, including by preventing lymphocyte activation and releasing cytokines in the bone microenvironment [54]. Research using animal models of postmenopausal osteoporosis has

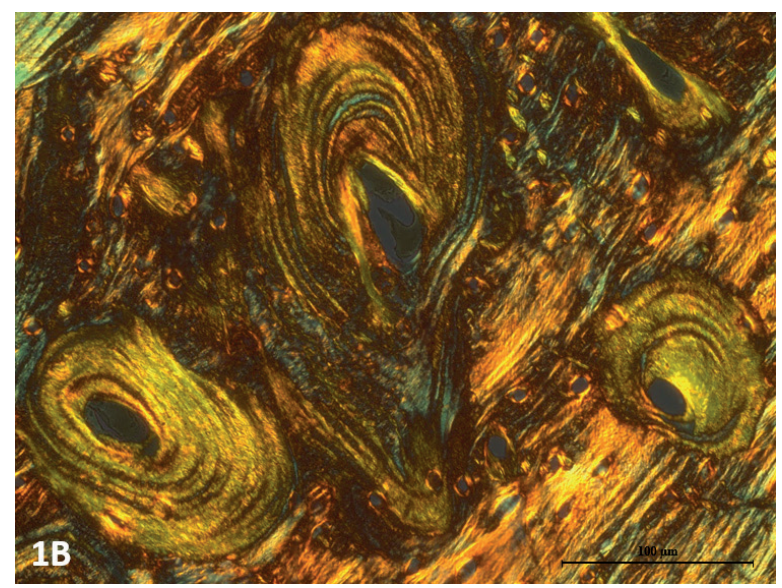

FIGURE 1. Bone sections stained with picrosirius red and imaged with polarized light. Organization of collagen fibers in the frontal bone of control (A) and experimental (B) rabbits treated with bLf, one month after an experimentally induced defect. The organization of the fiber appears less orderly, with abundant thin (yellow-greenish) collagen fibers in the control (A) and collagen fibers following osteonal organization with abundant yellow collagen fibers in the experimental specimens (B). Objective magnification $\times 40$ 
clearly indicated that supplementation with Lf can suppress osteoclast-mediated bone resorption and prevent bone loss. Lactoferin thus appears to be a promising candidate for the development of an anabolic therapeutic factor for osteoporosis [28].

As mentioned above, Lf appears to be a promising candidate as a therapeutic factor for osteoporosis [28]. Bharadway et al. investigated the benefits of supplementing with milk RNAse-enriched Lf (R-ELf) and calcium on the bone health of postmenopausal women, in comparison to a control group receiving only the calcium supplement. R-ELf significantly reduced bone resorption markers and simultaneously increased bone formation markers without any significant side effects. Additionally, R-ELf supplementation could achieve significant change in bone turnover markers within a short period, within only 3 months of supplementation [55]. More evidence of the beneficial role of Lf on bone health comes from experiments in postmenopausal animal models. Dietary Lf supplementation preserves bone mass and microarchitecture in ovariectomized rats, improving bone strength and bone mineral density [53]. Another study showed that oral bLf supplementation improved bone mineral density and femoral failure load of ovx mice in a dose-dependent manner [9].

Data obtained from in vivo studies support the osteogenic function of Lf and its practical application.

\section{APPLICATION OF LACTOFERRIN IN ORAL SURGERY AND DENTISTRY}

Lactoferrin, a protein with numerous antimicrobial, antiinflammatory, and osteogenic activity properties, has a range of applications in contemporary dentistry [4], including in disciplines such as dental surgery, periodontology, and pedodontics. Implantoprosthetics combines interdisciplinary knowledge in dental surgery and prosthodontics; the increasing frequency of implant therapy is associated with great benefits of this treatment for patients with missing teeth, providing stable support for prosthetic works and contributing to restoring stomatognathic system function, improving aesthetics, and eliminating the discomfort associated with removable prostheses. However, patients who qualify for implant therapy often lack the appropriate amount or quality of bone tissue in the site of the planned treatment.

Some dental implant procedures involve the application of short dental implants, bicortical implants, although the majority of patients prefer the procedure known as "two-stage placement", with the regeneration of lost bone tissue or enough bone density to hold the implant in place [56]. In first stage of the procedure, a bone graft is required, and autogenous grafts are the gold standard in bone regeneration [57]. Bone grafting can be done either before or during the implant procedure. It is not always possible to obtain an adequate amount of bone tissue from the patient during the autogenous graft, so xenomaterials are often used as an alternative [58]. In order to increase regenerative properties, the materials are linked with anabolic factors [59] such as Lf, which has documented anabolic properties towards bone tissue. In a study by Takaoka et al., the use of a gelatine carrier for Lf enabled a prolonged release of the glycoprotein to increase the activity of the osteoblasts and improve local bone regeneration [60]. However, in the study of Paknejad et al., Lf in combination with anorganic bovine bone $(\mathrm{ABB})$ was inserted into bone cavities but no statistically significant differences were observed between $A B B$ alone and LF+ABB in the bone regeneration [49]. Another study investigating the effect of Lf on bone resorption of a midpalatal suture during rapid expansion in rats showed that the bone:tissue volume ratio and the bone mineral density of the suture bone were significantly higher in expansion animals treated with Lf than in the expansion group without Lf. Moreover, the activity of OB-like cells and the amount of new bone formation were stimulated in the group treated with Lf, while the activity of the osteoclasts showed no significant differences between groups. These findings reveal that Lf can stimulate bone volume and bone density in midpalatal sutures during the suture remodeling process under tensile force, but that this effect is not caused by the reduction in bone resorption [61].

A gelatine microsphere (GM) carrier with bLf (GM-bLf) in combination with $\mathrm{ABB}$ was used in 1 study to regenerate bone tissue defects formed around dental implants in pigs [62]. This provided new bone formation at a higher percentage, as well as faster regeneration than in a group where only inorganic bovine material was implanted. Systemic Lf administration in bone defect regeneration conducted on animal models led to new bone growth in micro-CT images 2 weeks after the surgical procedure [50]. Oral administration of bLf was also demonstrated to have an advantageous effect on bone formation after the process of osteodistraction [51].

The use of Lf in implantology is also associated with its antiresorptive properties for titanium. Implant abutments are usually made of titanium alloy and are in contact with oral mucosa. An antimicrobial barrier should be created between the implant and the mucosa. This hermetic contact helps avoid the occurrence of peri-implantitis. Implant abutments covered with an Lf layer inhibited bacterial adhesion and showed antimicrobial activity. The use of Lf as a covering layer for titanium alloy elements is effective in avoiding peri-implantitis $[63,64]$.

According to Ishikado et al., systemic administration of liposomal bLf ( $\mathrm{L}$ bLf) inhibits the production of the inflammatory cytokines TNF- $\alpha$, IL-1 $\beta$, and IL- 6 in patients with periodontal disease. Liposomal bLf supplementation can therefore serve as an additional method of treatment for periodontitis [65]. Moreover, the measurements of Lf and $\alpha 1$-antitrypsin in gingival retention fluid can be used in the diagnosis of periodontal disease [66].

There is evidence that bLf naturally inhibits the adherence of Streptococcus mutans to enamel HA. Bovine Lf inhibits saliva-induced Streptococcus mutans aggregation. This action is due to the amino acid of Lf (Lf411). S. mutans is the main bacteria which causes tooth caries. A study by Oho et al. showed that the Lf domain that binds to salivary film lies in residues 473 to 538 . This region is also hidden by a disulfide bond formation 
between Cys481 and Cys532 in the Lf411 fragment. This part of Lf strongly inhibits the adhesion of S. mutans to saliva-covered HA beads $(\mathrm{p}<0.05)$. It was also reported that shorter fragments did not show significant adherence. Bovine Lf contains the antimicrobial domains lactoferricin B (Lfcin B) and lactoferrampin (Lfampin), which can exterminate bacteria and fungi, including C. albicans and E. coli [67].

Lactoferrin is also used as an ingredient in toothpastes which are intended for patients diagnosed with severe early childhood caries. It has been shown that the use of tooth paste with Lf, lysozyme, and lactoperoxidase significantly reduces the level of S. mutans and L. acidophilus in the saliva. A reduced level of microorganisms avoids disease progression [68]. Due to its multipotential properties, Lf can be successfully used in a variety of dental specialties.

\section{CONCLUSION}

Lactoferrin is a natural host protein with many biological functions. Lactoferrin promotes proliferation, growth, and the development of OBs. It also stimulates bone regeneration by inhibiting osteoclastogenesis and through antiapoptotic action. Moreover, Lf has antimicrobial and anti-inflammatory activity. All of these reasons mean that Lf has many applications in oral surgery.

\section{REFERENCES}

1. Adlerova L, Bartoskova A, Faldyna M. Lactoferrin: a review. Vet Med Czech 2008;53:457-68.

2. Garcia-Montoya IA, Cendón TS, Arévalo-Gallegos S, Rascón-Cruz Q. Lactoferrin a multiple bioactive protein: an overview. Biochim Biophys Acta 2012;1820(3):226-36

3. Kanwar JR, Roy K, Patel Y, Zhou SF, Singh MR, Singh D, et al. Multifunctional iron bound lactoferrin and na-nomedicinal approaches to enhance its bioactive functions. Molecules 2015;20:9703-31.

4. Trybek G, Metlerski M, Szumilas K, Aniko-Włodarczyk M, Preuss O, Grocholewicz K, et al. The biological properties of lactoferrin. Cent Eur J Sport Sci Med 2016;15(3):15-25.

5. Amini AA, Nair LS. Lactoferrin: a biologically active molecule for bone regeneration. Curr Med Chem 2011;18(8):1220-9.

6. Amini AA, Nair LS. Evaluation of the bioactivity of recombinant human lactoferrins toward murine osteob-last-like cells for bone tissue engineering. Tissue Eng Part A 2013;19(9-10):1047-55.

7. Ward PP, Paz E, Conneely OM. Multifunctional roles of lactoferrin: a critical overview. Cell Mol Life Sci 2005;62(22):2540-8.

8. Cornish J, Callon KE, Naot D, Palmano KP, Banovic T, Bava U, et al. Lactoferrin is a potent regulator of bone cell activity and increases bone formation in vivo. Endocrinology 2004;145(9):4366-74.

9. Blais A, Malet A, Mikogami T, Martin-Rouas C, Tomé D. Oral bovine lactoferrin improves bone status of ovariectomized mice. Am J Physiol Endocrinol Metab 2009;296(6):E1281-8.

10. Lorget F, Clough J, Oliveira M, Daury MC, Sabokbar A, Offord E. Lactoferrin reduces in vitro osteoclast differentiation and resorbing activity. Biochem Biophys Res Commun 2002;296(2):261-6.

11. Suzuki AY, Shin K, Lonnerdal B. Molecular cloning and functional expression of human intestinal lactofer-rin receptor. Biochemistry 2001;40(51):15771-9.

12. Lönnerdal B. Lactoferrin receptors in intestinal brush border membranes. Adv Exp Med Biol 1994;357:171-5.
13. Kuwata H, Yamauchi K, Teraguchi S, Ushida Y, Shimokawa Y, Toida T, et al. Functional fragments of inge-sted lactoferrin are resistant to proteolytic degradation in the gastrointestinal tract of adult rats. J Nutr 2001;131(8):2121-7.

14. Harada E, Itoh Y, Sitizyo K, Takeuchi T, Araki Y, Kitagawa H. Characteristic transport of lactoferrin from the intestinal lumen into the bile via the blood in piglets. Comp Biochem Physiol A Mol Integr Physiol 1999;124:321-7.

15. Ji ZS, Mahley RW. Lactoferrin binding to heparan sulfate proteoglycans and the LDL receptor related prote-in. Further evidence supporting the importance of direct binding of remnant lipoproteins to HSPG. Arterioscler Thromb 1994;14(12):2025-31.

16. Jenssen H, Andersen JH, Uhlin-Hansen L, Gutteberg TJ, Rekdal O. Anti-HSV activity of lactoferricin analogu-es is only partly related to their affinity for heparin sulfate. Antiviral Res 2004;61(12):101-9.

17. Legrand D, Vigie K, Said EA, Elass E, Masson M, Slomianny MC, et al. Surface nucleolin participates in both the binding and endocytosis of lactoferrin in target cells. Eur J Biochem 2004;271(2):303-17.

18. McAbee DD, Bennatt DJ, Ling YY. Identification and analysis of a CA(2+) -dependent Lf receptor in rat liver. Lf binds to the asialoglycoprotein receptor in a galactose-independent manner. Adv Exp Med Biol 1998;443:113-21.

19. Willnow TE, Goldstein JL, Orth K, Brown MS, Herz J. Low-density lipoprotein receptor-related protein and gp30 bind similar ligands, including plasminogen activator-inhibitor complexes and lactoferrin, and inhibi-tor of chylomicron remnant clearance. J Biol Chem 1992;267:26172-80.

20. Redwan EM, Uversky VN, El-Fakharany EM, Al-Mehdar H. Potential lactoferrin activity against pathogenic viruses. C R Biol 2014;337(10):581-95. doi: 10.1016/j.crvi.2014.08.003.

21. Frontera LS, Moyano S, Quassollo G, Lanfredi-Rangel A, Rópolo AS, Touz MC. Lactoferrin and lactoferricin endocytosis halt Giardia cell growth and prevent infective cyst production. Sci Rep. 2018 Dec 21;8(1):18020. doi: 10.1038/s41598-018-36563-1.

22. Grey A, Banovic T, Zhu Q, Watson M, Callon K, Palmano K, et al. The lowdensity lipoprotein receptor-related protein 1 is a mitogenic receptor for lactoferrin in osteoblastic cells. Mol Endocrinol 2004;18(9):2268-78.

23. Grey A, Zhu Q, Watson M, Callon K, Cornish J. Lactoferrin potently inhibits osteoblast apoptosis, via an LRP1-independent pathway. Mol Cell Endocrinol 2006;251(1-2):96-102.

24. Hou JM, Chen EY, Lin F, Lin QM, Xue Y, Lan XH, et al. Lactoferrin Induces Osteoblast Growth through IGF-1R. Int J Endocrinol 2015;2015:282806.

25. Massey HM, Flanagan AM. Human osteoclasts derive from CD14-positive monocytes. Br J Haematol 1999;106(1):167-70.

26. Gay CV, Weber JA. Regulation of differentiated osteoclasts. Rev Eukaryot Gene Expr 2000;10:213-30.

27. Boyce BF. Advances in the regulation of osteoclasts and osteoclast functions. J Dent Res 2013;92(10):860-7.

28. Cornish J, Naot D. Lactoferrin as an effector molecule in the skeleton. Biometals 2010;23(4):425-30.

29. Legrand D, Elass E, Carpentier M, Mazurier J. Interaction of lactoferrin with cells involved in immune func-tion. Biochem Cell Biol 2006;84(3):282-90.

30. Karsenty G, Wagner EF. Reaching a genetic and molecular understanding of skeletal development. Dev Cell 2002;2(4):389-406.

31. Yamaguchi A. Regulation of differentiation pathway of skeletal mesenchymal cells in cell lines by trans-forming growth factor-beta superfamily. Semin Cell Biol 1995;6(3):165-73.

32. Yagi M, Suzuki N, Takayama T, Arisue M, Kodama T, Yoda Y, et al. Effects of lactoferrin on the differentia-tion of pluripotent mesenchymal cells. Cell Biol Int 2009;33:283-9.

33. Zhang JL, Han X, Shan YJ, Zhang LW, Du M, Liu M, et al. Effect of bovine lactoferrin and human lactoferrin on the proliferative activity of the osteoblast cell line MC3T3-E1 in vitro. J Dairy Sci 2018;101(3):1827-33. doi: 10.3168/jds.2017-13161.

34. Liu M, Fan F, Shi P, Tu M, Yu C, Yu C, et al. Lactoferrin promotes MC3T3E1 osteoblast cells proliferation via MAPK signaling pathways. Int J Biol Macromol 2018;107(Pt A):137-43. doi: 10.1016/j.ijbiomac.2017.08.151.

35. Montesi M, Panseri S, Iafisco M, Adamiano A, Tampieri A. Effect of hydroxyapatite nanocrystals functiona-lized with lactoferrin in osteogenic differentiation of mesenchymal stem cells. J Biomed Mater Res A 2015;103(1):224-34. 
36. Montesi M, Panseri S, Iafisco M, Adamiano A, Tampieri A. Coupling Hydroxyapatite Nanocrystals with Lac-toferrin as a Promising Strategy to Fine Regulate Bone Homeostasis. PLoS One 2015;10(7):e0132633.

37. Vandrovcova M, Douglas TE, Heinemann S, Scharnweber D, Dubruel P, Bacakova L. Collagen-lactoferrin fi-brillar coatings enhance osteoblast proliferation and differentiation. J Biomed Mater Res A 2015;103(2):525-33.

38. Hesse E, Hefferan TE, Tarara JE, Haasper C, Meller R, Krettek C, et al. Col lagen type I hydrogel allows migra-tion, proliferation, and osteogenic differentiation of rat bone marrow stromal cells. J Biomed Mater Res A 2010;94(2):442-9.

39. Masi L, Franchi A, Santucci M, Danielli D, Arganini L, Giannone V, et al. Adhesion, growth, and matrix pro-duction by osteoblasts on collagen substrata. Calcif Tissue Int 1992;51(3):202-12.

40. Matthews BG, Naot D, Callon KE, Musson DS, Locklin R, Hulley PA, et al. Enhanced osteoblastogenesis in three-dimensional collagen gels. Bonekey Rep 2014;3:560.

41. Takayama Y, Mizumachi K. Effect of bovine lactoferrin on extracellular matrix calcification by human oste-oblast-like cells. Biosci Biotechnol Biochem 2008;72(1):226-30.

42. Takayama Y, Mizumachi K. Effect of lactoferrin-embedded collagen mem brane on osteogenic differentia-tion of human osteoblast-like cells. J Biosci Bioeng 2009;107(2):191-5

43. Huang N, Bethell D, Card C, Cornish J, Marchbank T, Wyatt D, et al. Bioactive recombinant human lactofer-rin, derived from rice, stimulates mammalian cell growth. In Vitro Cell Dev Biol Anim 2008;44(10):464-71.

44. Nandi S, Yalda D, Lu S, Nikolov Z, Misaki R, Fujiyama K, et al. Process development and economic evalua-tion of recombinant human lactoferrin expressed in rice grain. Transgenic Res 2005;14:237-49.

45. Ward PP, Uribe-Luna S, Conneely OM. Lactoferrin and host defense. Biochem Cell Biol 2002;80(1):95-102.

46. Häkkinen L, Uitto VJ, Larjava $\mathrm{H}$. Cell biology of gingival wound healing. Periodontol 2000 2000;24:127-52

47. Proksch S, Steinberg T, Keller C, Wolkewitz M, Wiedmann-Al-Ahmad M Finkenzeller G, et al. Human saliva exposure modulates bone cell performance in vitro. Clin Oral Invest 2012;16:69-77.

48. Paknejad M, Rokn A, Sabur A, Elhami F. Histologic and histomorphometric study on the effects of lactofer-rin and porous bovine bone mineral (Bio-Oss) on the regeneration of bone defects made on rabbit calva-rium J Dent Med 2010;23:167-74.

49. Paknejad M, Rokn AR, Yaraghi AA, Elhami F, Kharazifard MJ, Moslemi N. Histologic and histomorphome-tric evaluation of the effect of lactoferrin combined with anorganic bovine bone on healing of experimental-ly induced bony defects on rabbit calvaria. Dent Res J (Isfahan) 2012;9(1):75-80.

50. Yoshimaki T, Sato S, Tsunori K, Shino H, Iguchi S, Arai Y, et al. Bone regeneration with systemic administra-tion of lactoferrin in non-critical-sized rat calvarial bone defects. J Oral Sci 2013;55(4):343-8.

51. Li W, Zhu S, Hu J. Bone Regeneration Is Promoted by Orally Administered Bovine lactoferrin in a Rabbit Ti-bial Distraction Osteogenesis Model Clin Orthop Relat Res 2015;473(7):2383-93.

52. Hou JM, Xue Y, Lin QM. Bovine lactoferrin improves bone mass and microstructure in ovariectomized rats via OPG/RANKL/RANK pathway. Acta Pharmacol Sin 2012;33(10):1277-84
53. Guo HY, Jiang L, Ibrahim SA, Zhang L, Zhang H, Zhang M, et al. Orally administered lactoferrin preserves bone mass and microarchitecture in ovariectomized rats. J Nutr 2009;139(5):958-64.

54. Malet A, Bournaud E, Lan A, Mikogami T, Tomé D, Blais A. Bovine lactoferrin improves bone status of ova-riectomized mice via immune function modulation. Bone 2011;48(5):1028-35.

55. Bharadway S, Naidu AGT, Betageri GV, Prasadarao NV, Naidu AS. Milk ribonuclease-enriched lactoferrin in-duces positive effects on bone turnover markers in postmenopausal women. Osteoporos Int 2009;20(9):1603-11.

56. Misch CE, Dietsh F. Bone-grafting materials in implant dentistry. Implant Dent 1993;2(3):158-67.

57. Stern A, Barzani G. Autogenous bone harvest for implant reconstruction. Dent Clin North Am 2015;59(2):409-20.

58. Botticelli D, Berglundh T, Lindhe J. The influence of a biomaterial on the closure of a marginal hard tissue defect adjacent to implants. An experimental study in the dog. Clin Oral Implants Res 2004;15(3):285-92.

59. Jung RE, Glauser R, Schärer P, Hämmerle CHF, Sailer HF, Weber FE. Effect of rhBMP-2 on guided bone rege-neration in humans. A randomized, controlled clinical and histomorphometric study. Clin Oral Implants Res 2003;14(5):556-68

60. Takaoka R, Hikasa Y, Hayashi K, Tabata Y. Bone regeneration by lactoferrin released from a gelatin hydro-gel. J Biomater Sci Polym Ed 2011;22(12):1581-9.

61. Cheng Y, Sun J, Zhou Z, Pan J, Zou S, Chen J. Effects of lactoferrin on bone resorption of midpalatal suture during rapid expansion in rats. Am J Orthod Dentofacial Orthop 2018;154(1):115-127.

62. Görmez U, Kürkcü M, E Benlidayi M, Ulubayram K, Sertdemir Y, Dağlioğlu K. Effects of bovine lactoferrin in surgically created bone defects on bone regeneration around implants. J Oral Sci 2015;57(1):7-15.

63. Yoshida E, Hayakawa T. Adsorption Analysis of Lactoferrin to Titanium, Stainless Steel, Zirconia, and Polymethyl Methacrylate Using the Quartz Crystal Microbalance Method. Biomed Res Int 2016;2016:3961286.

64. Nagano-Takebe F, Miyakawa H, Nakazawa F, Endo K. Inhibition of initial bacterial adhesion on titanium surfaces by lactoferrin coating. Biointerphases 2014;9(2):029006.

65. Ishikado A, Uesaki S, Suido H, Nomura Y, Sumikawa K, Maeda M, et al. Human trial of liposomal lactoferrin supplementation for periodontal disease. Biol Pharm Bull 2010;33:1758-62.

66. Koshi R, Kotani K, Ohtsu M, Yoshinuma N, Sugano N. Application of Lactoferrin and $\alpha 1$-antitrypsin gingiva; retention fluid to diagnosis of periodontal disease. Dis Markers 2018:2018:4308291.

67. Oho T, Mitoma M, Koga T. Functional domain bovine milk lactoferrin which inhibits the adherence of Streptococcus mutans cells to a salivary film. Infect Immun 2002;70(90):5279-82.

68. Gudipaneni RK, Kumar RV, GJ, Peddengatagari S, Duddu Y. Short term comparative evaluation of antimicro-bial efficacy of tooth paste containing lactoferrin, lysozyme, lactoperoxidase in children with severe early childhood caries: a clinical study. J Clin Diagn Res 2014;8(4):ZC18-20. 